\title{
The effect of a short ultrasound training course on the performance of emergency medical technicians in terms of using extended-focused assessment with sonography in trauma (E-FAST) in the pre-hospital setting
}

\author{
Anita Sabzghabaei $^{\circledR}{ }^{\circledR}$ Fariba Farahi $^{\circledR}$, Masoud Ghanbari Boroujeni ${ }^{\circledR}$, Hamidreza Hatamabadi2 ${ }^{*}$ \\ 'Department of Emergency Medicine, Shahid Beheshti University of Medical Science, Tehran, Iran \\ 2Safety Promotion and Injury Prevention Research Center, Shahid Beheshti University of Medical Sciences, Tehran. Iran
}

\author{
Received: 3 December 2020 \\ Accepted: 18 February 2021 \\ Published online: 9 March 2021 \\ *Corresponding author: \\ Hamidreza Hatamabadi, MD, Safety \\ Promotion and Injury Prevention \\ Research Center, Shahid Beheshti \\ University of Medical Sciences, \\ Tehran. Iran. P.O. Box: 193955487, \\ Tel: +98-21-73432389, Fax: +98-21- \\ 77557069,Email: hhatamabadi@ \\ yahoo.com
}

Competing interests: None.

Funding information: This research was supported financially by the Safety Promotion and Injury Prevention Research Center, Shahid Beheshti University of Medical Sciences, Tehran, Iran (Grant number: 7863)

Citation: Sabzghabaei A, Farahi F Ghanbari Boroujeni M, Hatamabadi $H$. The effect of a short ultrasound training course on the performance of emergency medical technicians in terms of using extended-focused assessment with sonography in trauma (e-fast) in the pre-hospital setting. Journal of Emergency Practice and Trauma 2021; 7(2): 97100. doi: 10.34172/jept.2021.09

\begin{abstract}
Objective: The aim of this study was to determine the effect of an ultrasound training course on the performance of emergency medical technicians (EMTs) in terms of using extended-focused assessment with sonography in trauma (E-FAST) in the pre-hospital setting.

Methods: In this quasi-experimental study, 107 EMTs affiliated to Shahid Beheshti and Kashan Universities of Medical Sciences, were randomly selected. At first, the EMTs were given a pre-test of five images concerning normal and abnormal ultrasound views of thoracic and abdominal cavities. Then, 6 hours of theoretical and practical sessions were done by emergency medicine specialists to teach the EMTs to use ultrasound for the detection of pneumothorax and free fluid. The EMTs were tested again in the form of 18 images post-test as well as a 13-item survey to evaluate their skills in taking correct 10 E-FAST.

Results: A total of 107 EMTs with the mean age of $27 \pm 15.21$ and average of 3.5 years work experience participated in this study. The mean of test score increased from $0.57 \pm 0.19$ to $0.81 \pm 0.08$ (with $P<0.001$ ) out of the total score of 100 . In the 4 -graded survey, $98 \%$ of EMTs obtained good to excellent grades in working with ultrasound machine. Also, 50.5\% of EMTs were good in taking Morison's view and $46.6 \%$ and $45.6 \%$ had medium grades in pericardial and splenorenal views, respectively. In addition, $22.3 \%$ had weak grades in taking pericardial views.

Conclusion: The results of the present study showed that the implementation of the training course significantly improved the accuracy of free fluid detection in thoracic and abdominal cavities by EMTs.

Keywords: Training, Ultrasonography, Pre-hospital setting, Emergency medical Technicians, E-FAST, Free fluid, Thoracic and abdominal injury
\end{abstract}

\section{Introduction}

Emergency medical services (EMS) technicians play an important role in preventing diagnostic errors and welltimed management of patients as they have the first contact with trauma patients in conditions like disaster and military medicine (1-3). Some believe that up to $30 \%$ of deaths from trauma in the United States can be prevented, and failing to recognize intra-abdominal injuries following blunt traumas to the abdomen is a common cause of preventable deaths $(4,5)$. Diagnosing pneumothorax in critical traumatic patients can save patients' lives (6).

Since the clinical examination is not reliable for the correct evaluation of traumatized patients, emergency ultrasound technique can be used in accidents and deaths in order to evaluate and manage patients quickly and provide diagnostic and procedural guidance (7). The most important clinical application of ultrasound is the use of the basic data to guide the triage and manage patients in a critical condition and may improve patient management $(8,9)$. Therefore, similar to the urgent need of EMS for equipping ambulances with electrocardiogram 
and training the personnel for the early diagnosis of myocardial infarction with the high ST segment, the availability of portable ultrasound units in ambulances and the development of skills in the personnel to detect free air and fluid in the thoracic and abdominal cavities can activate the trauma team through faster diagnosis and notification of the trauma center, improving the prognosis of the trauma patients (10-12).

However, the cost of equipment and education is the most important barrier in the implementation of the ultrasound technique, and most of the medical managers require evidence before implementation because they want to make sure that pre-hospital ultrasound improves the patients' outcome (10). In this regard, in addition to portable and multipurpose equipment, there is a need for trained personnel, specific protocols and preparation, and there are still some challenges.

In Iran, ultrasound has not yet been used in EMS ambulances. Officials believe that its use in the ambulance is costly and of no use. Given the importance of early detection of free fluid in the thoracic and abdominal cavities in reducing the mortality of traumatic patients, we decided to investigate the impact of an ultrasound training course on the performance of technicians to detect free air and fluid in the thorax and abdomen.

\section{Methods}

This quasi-experimental study was conducted in Shahid Beheshti and Kashan Universities of Medical Sciences of Iran. Informed consent was obtained from all participants and the training workshops were held in the universities separately. Participants did not get prior ultrasonography training. During the study, a portable ultrasound SonoSite (Bothell, WA) M-Turbo with L25n 13-6-MHz linear and $\mathrm{P} 21 \mathrm{n}$ 5-1-MHz phased-array transducers unit was used.

After explaining the importance of the issue and the method of the study to all emergency medical technicians (EMTs) of the two universities, 107 EMTs were randomly selected and enrolled into the study (60 participants from Shahid Beheshti University of Medical Sciences and 47 participants from Kashan University of Medical Sciences). The sample size for EMTs of each university was calculated based on Cochran's sample size formula with the confidence interval of $90 \%$. The process of randomization was conducted through a computer-based approach.

In the first phase of the study, the emergency technicians were given a pre-test of five images regarding normal and abnormal ultrasound views of thoracic and abdominal cavities for determining the general knowledge of EMS technicians on the detection of free fluid in the abdomen, thoracic cavities, and pericardium. The pre-test score was calculated for each participant.

After training workshops, participants were tested again in the form of 18 images post-test as well as a 10-item OSCE (Objective structured clinical examination) exam to evaluate their skills in taking correct extended-focused assessment with sonography in trauma (E-FAST) views. Post-test contained the same questions from the pre-test, and other subjects were not given answers or feedback on their initial tests. A checklist consisting of 13 questions was provided to evaluate using probe and adequacy of thoracic and abdominal views. In this 4-graded survey, "excellent" refers to the performing each step without any mistake and in case of one mistake in each step, the score would be "good" for that step. We used this checklist to assess the EMTs' skills in operating the equipment correctly, selecting the appropriate probe, and locating the probe in five abdominal regions correctly.

Both pre-and post-tests were validated and confirmed by emergency medicine specialists and ultrasonography images were originally obtained in the Emergency Department setting by emergency specialists. The posttests were conducted after 2 hours and one day in Tehran and Kashan Universities of Medical Sciences, respectively.

Finally, the technicians' learning and its score was defined as the percentage of correct answers out of the total number of questions (converting to 100 points), indicating the effectiveness and efficacy of training the technicians.

In the second phase of the study, 6 hours of theoretical and practical sessions were done by emergency medicine specialists to teach participants how to grip the ultrasound probe in hand, achieve the desired views and use ultrasound for the detection of free fluid in the abdominal and thoracic cavities in bedside ultrasonography on a human model.

During the training, pictures of negative and normal cases were presented, then the positive ones. In handson workshops, there were formal instructions on probe orientation, adequacy of views of six anatomical areas of the thoracic and abdominal cavities, and important considerations to diagnose pneumothorax and free fluid in the thoracic and abdominal.

Data were analyzed by using SPSS software version 21 (IBM Corporation, Armonk, NY). Demographic variables were analyzed using descriptive statistics. Chi-square and paired sample $t$ tests were used for their correlation. Statistical significance was set at $P<0.05$.

\section{Results}

From 107 EMTs participating in the study, four did not take the OSCE exam, and were excluded from the study. Consequently, 103 participants (96\%) entered the study. The mean of participants' age was $27 \pm 15.21$ years and all of them were males.

Comparing the pre-test and post-test results, the mean of test score increased from $57 \pm 19$ to $81 \pm 8$ ( $P$ value< 0.001). In Kashan EMTs group, scores increased from 62 \pm 21 to $83 \pm 8(P$ value $<0.001)$ and in Tehran EMTs group, scores increased from $52 \pm 16$ to $80 \pm 8$ ( $P$ value $<0.001)$

The percentages of correct responses in the post-test 
of the two groups showed no significant difference in correct answers $(P=0.13)$, and the scores of both groups increased significantly. The mean percentage of post-test correct answers was $83 \%$. The results showed a significant difference between the results of the pre-and post-test as shown in Table $1(P<0.0001)$.

As depicted in Table 2, the pre-test result in Kashan university was higher (62\% versus $52 \%$ in Tehran university); possibly due to their prior reading and interest.

The study checklist was graded as weak, medium, good and excellent. In this 4-graded survey, ninety eight percent of EMTs obtained good to excellent grades in working with ultrasound machine. Also, 50.5\% were good in taking Morison's view and $46.6 \%$ and $45.6 \%$ had medium grades in pericardial and splenorenal views, respectively. Besides, $22.3 \%$ had weak grades in taking pericardial views. Table 3 shows the results of the evaluation of participants after the implementation of training (study checklist).

\section{Discussion}

According to the results of this study, training significantly increased the diagnostic accuracy and skills of emergency technicians concerning pneumothorax and free fluid of the thoracic and abdominal cavities by ultrasound in patients with blunt abdominal traumas. The majority of the participants got good scores in taking Morison's view. However, in terms of probe location in the space between the spleen and the left kidney as well as pericardial fluid, majority of the participants achieved moderate scores. We only observed high scores for their views regarding the space around the bladder. Conversely, low scores were obtained regarding their views for pericardial fluid and the space between the spleen and the left kidney.

Walcher et al. compared the accuracy of abdominal ultrasound diagnosis done by three groups of medical staffs consisting the experienced group, the untrained control group and the participants in one-day training course for abdominal ultrasound in traumatic patients. After completing the one-day P-FAST course, participants were able to perform ultrasound procedures with a high accuracy rate in the accident scene (12). In addition, the results of a study by Noble et al similarly showed that holding a short training session significantly improved physicians' diagnostic skills in relation to

Table 1. Comparison of pre- and post- test results in the detection of negative and positive images

\begin{tabular}{lccccc}
\hline Test & $\mathbf{n}$ & Mean & SD & t & df \\
\hline Percentage of correct response (pre-test) & 103 & 57 & 19 & 4.75 \\
Percentage of correct response (post-test) & 103 & 81 & 8 & 0.0001 \\
\hline
\end{tabular}

Table 2. Comparison of pre and post -test results in the detection of negative and positive images separately in the two groups

\begin{tabular}{lllcc}
\hline Group & Test & n & Mean & SD \\
\hline \multirow{2}{*}{ Kashan EMTs } & Percentage of correct response (pre-test) & 47 & 62 & 21 \\
& Percentage of correct response (post-test) & 47 & 83 & 0.00 \\
\multirow{2}{*}{ Tehran EMT s } & Percentage of correct response (pre-test) & 56 & 52 & 16 \\
& Percentage of correct response (post-test) & 56 & 80 & 8.00 \\
\hline
\end{tabular}

Table 3. Evaluation of the participants after the implementation of the training plan

\begin{tabular}{|c|c|c|c|c|c|c|}
\hline Questions & Excellent $(\%)$ & Good $(\%)$ & $\operatorname{Mod}(\%)$ & Poor $(\%)$ & $\chi^{2}$ & $P$ \\
\hline Did you learn how to correctly use the equipment? & 1.9 & 96.1 & 1.9 & 0 & 5.24 & 0.01 \\
\hline Did you select a proper probe for examining the abdomen? & 3.9 & 96.1 & 0 & 0 & 4.96 & 0.01 \\
\hline Did you select a proper probe for examining the blood vessels? & 1.9 & 95.1 & 2.9 & 0 & 6.68 & 0.01 \\
\hline Was the probe placed correctly in relation to the Morrison's pouch? & 2.9 & 90.3 & 6.8 & 0 & 4.91 & 0.01 \\
\hline Was the probe placed correctly between spleen and left kidney? & 1.9 & 34 & 62.1 & 1.9 & 7.21 & 0.01 \\
\hline Was the probe placed correctly around the bladder? & 35.9 & 47.6 & 16.1 & 0 & 6.20 & 0.01 \\
\hline Was the probe placed correctly beneath the sternum? & 19.4 & 49.5 & 28.2 & 2.9 & 7.54 & 0.01 \\
\hline Was the probe placed correctly to determine the peripheral vessels? & 1.9 & 78.6 & 19.4 & 0 & 6.39 & 0.01 \\
\hline Did you achieve the appropriate view for the Morrison's pouch? & 6.8 & 50.5 & 39.8 & 2.9 & 4.32 & 0.01 \\
\hline Did you achieve the appropriate view for the area between spleen and left kidney? & 2.9 & 32 & 45.6 & 19.4 & 3.98 & 0.01 \\
\hline Did you achieve the appropriate view for the area around the bladder? & 48.5 & 32 & 16.5 & 1.9 & 4.25 & 0.01 \\
\hline Did you achieve the appropriate view for the pericardial fluid? & 4.9 & 26.2 & 46.6 & 22.3 & 6.08 & 0.01 \\
\hline Did you achieve the appropriate view for lung? & 1 & 50.5 & 43.7 & 4.9 & 7.54 & 0.01 \\
\hline
\end{tabular}


pneumothorax and pulmonary edema in both with and without ultrasound experience (13). Heegaard et al assessed the ability of paramedics trained in ultrasound in performing and interpreting FAST exams in the prehospital care environment. Finally, a 100\% success rate of FAST interpretation was observed among emergency technicians (11).

Based on the narrative review conducted by Ketelaars et al, pre-hospital ultrasound improves the pre-hospital health-care providers' capacity in diagnosis and may result in better treatment decisions, transport mode and destination, but pre-hospital caregivers should be aware of pre-hospital ultrasound limitations as its accuracy depends on the experience and skill of the performer (7). The most important challenge is the impact of cost on the EMS organizations, training, repair and maintenance, and the challenges related to the technical aspects of the ultrasound technique (8). Taylor et al also reported that the cost of equipment and education is the most important barrier for the implementation of the ultrasound technique in North America, and most of the medical managers require evidence before implementation because they want to make sure that pre-hospital ultrasound improves the patients' outcomes (10).

\section{Conclusion}

According to the results of this study and similar researches, ultrasound can be used in the pre-hospital setting to improve management of patients and may develop the diagnosis and treatment in emergency medicine. However, this requires portable equipment, trained personnel, and specific protocols. Training improves the accuracy of ultrasound performed by the emergency medical technicians in the pre-hospital environment.

\section{Ethical Issues}

This study was approved by the research ethics committee of Shahid Beheshti University of Medical Sciences and the ethical code number is IR.SBMU.MSP.REC.1396.652. Informed consent was obtained from all participants.

\section{Authors' contributions}

AS designed the study, collected all data, wrote the manuscript; FF contributed in the manuscript writing; MGB helped with data collection, $\mathrm{HH}$ collected data and gained ethical approval. All authors reviewed and edited the manuscript and approved the final version of the manuscript.

\section{References}

1. Bost N, Crilly J, Wallis M, Patterson E, Chaboyer W.
Clinical handover of patients arriving by ambulance to the emergency department-a literature review. Int Emerg Nurs 2010; 18(4): 210-20. doi: 10.1016/j.ienj.2009.11.006.

2. Walcher F, Weinlich M, Conrad G, Schweigkofler U, Breitkreutz R, Kirschning T, et al. Prehospital ultrasound imaging improves management of abdominal trauma. $\mathrm{Br} \mathrm{J}$ Surg 2006; 93(2): 238-42. doi: 10.1002/bjs.5213.

3. Heegaard W, Plummer D, Dries D, Frascone RJ, Pippert G, Steel D, et al. Ultrasound for the air medical clinician. Air Med J 2004; 23(2): 20-3. doi: 10.1016/j.amj.2003.12.006.

4. Richards JR, Schleper NH, Woo BD, Bohnen PA, McGahan JP. Sonographic assessment of blunt abdominal trauma: a 4-year prospective study. J Clin Ultrasound 2002; 30(2): 5967. doi: 10.1002/jcu.10033.

5. Poletti PA, Kinkel K, Vermeulen B, Irmay F, Unger PF, Terrier F. Blunt abdominal trauma: should US be used to detect both free fluid and organ injuries? Radiology 2003; 227(1): 95-103. doi: 10.1148/radiol.2271020139.

6. Husain LF, Hagopian L, Wayman D, Baker WE, Carmody KA. Sonographic diagnosis of pneumothorax. J Emerg Trauma Shock 2012; 5(1): 76-81. doi: 10.4103/09742700.93116.

7. Ketelaars R, Reijnders G, van Geffen GJ, Scheffer GJ, Hoogerwerf N. ABCDE of prehospital ultrasonography: a narrative review. Crit Ultrasound J 2018; 10(1): 17. doi: 10.1186/s13089-018-0099-y.

8. El Zahran T, El Sayed MJ. Prehospital ultrasound in trauma: a review of current and potential future clinical applications. J Emerg Trauma Shock 2018; 11(1): 4-9. doi: 10.4103/jets. jets_117_17.

9. Rudolph SS, Sørensen MK, Svane C, Hesselfeldt R, Steinmetz J. Effect of prehospital ultrasound on clinical outcomes of non-trauma patients--a systematic review. Resuscitation 2014; 85(1): 21-30. doi: 10.1016/j.resuscitation.2013.09.012.

10. Taylor J, McLaughlin K, McRae A, Lang E, Anton A. Use of prehospital ultrasound in North America: a survey of emergency medical services medical directors. BMC Emerg Med 2014; 14: 6. doi: 10.1186/1471-227x-14-6.

11. Heegaard W, Hildebrandt D, Spear D, Chason K, Nelson B, Ho J. Prehospital ultrasound by paramedics: results of field trial. Acad Emerg Med 2010; 17(6): 624-30. doi: 10.1111/j.1553-2712.2010.00755.x.

12. Walcher F, Kirschning T, Müller MP, Byhahn C, Stier $\mathrm{M}$, Rüsseler $\mathrm{M}$, et al. Accuracy of prehospital focused abdominal sonography for trauma after a 1-day handson training course. Emerg Med J 2010; 27(5): 345-9. doi: 10.1136/emj.2008.059626.

13. Noble VE, Lamhaut L, Capp R, Bosson N, Liteplo A, Marx JS, et al. Evaluation of a thoracic ultrasound training module for the detection of pneumothorax and pulmonary edema by prehospital physician care providers. BMC Med Educ 2009; 9: 3. doi: 10.1186/1472-6920-9-3. 